\title{
Nuclear shadowing in DIS in future electron-ion colliders
}

\author{
Michal Krelina $^{a, *}$ and Jan Nemchik ${ }^{a, b}$ \\ ${ }^{a}$ Czech Technical University in Prague, FNSPE, \\ Břehová 7, 11519 Prague, Czech Republic \\ ${ }^{b}$ Institute of Experimental Physics SAS, \\ Watsonova 47, 04001 Košice, Slovakia \\ E-mail: michal.krelina@cvut.cz, nemcik@saske.sk
}

We present a comprehensive study of shadowing effects in deep-inelastic scattering off nuclei in kinematic regions accessible by future experiments at electron-ion colliders. The calculations of shadowing are performed within the color dipole formalism using a rigorous Green function technique. This allows incorporating naturally the effects of quantum coherence and color transparency, which are not consistently included in present calculations. We analyze as well the magnitude of gluon shadowing representing the shadowing correction coming from higher Fock states of the photon containing gluons. We present for the first time the impact parameter behavior of shadowing effects and analyze the theoretical uncertainties in their estimations using different models for the dipole cross section. Our predictions for the onset of gluon shadowing corrections at small Bjorken $x_{B j}$ can be verified by future experiments at electron-ion colliders.

40th International Conference on High Energy physics - ICHEP2020

July 28 - August 6, 2020

Prague, Czech Republic (virtual meeting)

\footnotetext{
${ }^{*}$ Speaker
} 


\section{Introduction}

The future Electron-Ion colliders (EICs) [1,2] will represent an effective tool for precise study of the nuclear structure, as well as QCD aspects of various processes and effects occurring in interactions with nuclei. One of the main topics will concern to nuclear shadowing at small Bjorken $x_{B j}$, which is best studied in nuclear deep inelastic scattering (nDIS) process. Particularly, the usually used and experimentally measured observable for the study of shadowing phenomena in DIS is the nuclear structure function $F_{2}^{A}$. In the region of small $x_{B j}$ the effect of nuclear shadowing manifests itself as an inequality $F_{2}^{A} /\left(A F_{2}^{N}\right)<1$, where $A$ is the number of nucleons in a nuclear target, and $F_{2}^{N}$ represents the free nucleon structure function. For the nuclear shadowing topic overview, see [3].

The precise knowledge and understanding of shadowing effects is crucial for a correct theoretical description and interpretation of various processes off nuclei in the region of small Bjorken $x_{B j} \lesssim 0.01$, such as diffractive photo- and electroproduction of vector mesons, Drell-Yan process, direct photon production, semi-inclusive production of hadrons, etc.

In this report, we will treat the nuclear shadowing within the light-front (LF) color dipole formalism. Within the LF formalism, the nuclear target is in the rest frame, and the incoming colliding object can be seen as a superposition of various Fock components with different lifetimes related to their effective masses. This means that the incoming virtual photon will fluctuate preferably into a $q \bar{q}$ Fock state at smaller energies with a gradual manifestation of multi-gluon Fock fluctuations ( $q \bar{q} G, q \bar{q} 2 G$, etc.) at smaller $x_{B j}$. Consequently, the dynamics of the nuclear shadowing is controlled by the lifetime of such fluctuations which is also known as the coherence time or length (CL). For the lowest $|q \bar{q}\rangle$ Fock state the CL reads,

$$
l_{c}=\frac{2 v}{Q^{2}+M_{q \bar{q}}^{2}},
$$

where $v$ and $Q^{2}$ are the photon energy and virtuality, and $M_{q \bar{q}}$ is the effective mass of the $q \bar{q}$ pair.

For the maximal onset of shadowing effects, the CL should considerably exceed the nuclear radius $R_{A}, l_{c} \gg R_{A}$. Otherwise, the destructive interference of amplitudes corresponding to interactions which occur on different bound nucleons, is reduced due to a shorter lifetime of the $|q \bar{q}\rangle$ Fock state, causing weaker shadowing effects. In the present paper, we include effects of quantum coherence properly with no restrictions for CL using a rigorous path integral technique [3]. The same formalism is also used for calculations of shadowing corrections from higher multigluon Fock components of the photon. In comparison with the photon $q \bar{q}$ fluctuations, they have shorter lifetimes due to their larger effective masses, and the corresponding proper manifestation of shadowing effects requires larger photon energies. In the next Section, we present a brief review of the color dipole formalism as well as an outline of the Green function technique controlling the nuclear shadowing dynamics as a function of CL. The corresponding results of nuclear shadowing are in Sect. 3 and conclusions in Sect. 4.

\section{Nuclear shadowing within the color dipole formalism}

In the rest frame of the nucleus the photo-nuclear cross section reads as follows:

$$
\sigma_{\text {tot }}^{\gamma^{*} A}\left(x_{B j}, Q^{2}\right)=A \sigma_{\text {tot }}^{\gamma^{*} N}\left(x_{B j}, Q^{2}\right)-\Delta \sigma_{t o t}\left(x_{B j}, Q^{2}\right),
$$


where $\Delta \sigma_{t o t}\left(x_{B j}, Q^{2}\right)$ represents the summation of shadowing corrections coming from different Fock states, $|q \bar{q}\rangle,|q \bar{q} G\rangle,|q \bar{q} 2 G\rangle, \cdots$

$$
\Delta \sigma_{t o t}\left(x_{B j}, Q^{2}\right)=\Delta \sigma_{t o t}^{q \bar{q}}\left(x_{B j}, Q^{2}\right)+\Delta \sigma_{t o t}^{q \bar{q} G}\left(x_{B j}, Q^{2}\right)+\Delta \sigma_{t o t}^{q \bar{q} 2 G}\left(x_{B j}, Q^{2}\right)+\cdots .
$$

Within the color dipole approach, the variable $\sigma_{t o t}^{\gamma^{*} N}\left(x_{B j}, Q^{2}\right)$ can be expressed in the quantummechanical form,

$$
\sigma_{\text {tot }}^{\gamma^{*} N}\left(x_{B j}, Q^{2}\right)=\left\langle\Psi_{q \bar{q}}(r, \alpha)\left|\sigma_{q \bar{q}}(r)\right| \Psi_{q \bar{q}}(r, \alpha)\right\rangle=\int d^{2} r \int_{0}^{1} d \alpha\left|\Psi_{q \bar{q}}\left(\vec{r}, \alpha, Q^{2}\right)\right|^{2} \sigma_{q \bar{q}}(\vec{r}, s),
$$

where $\Psi_{q \bar{q}}\left(\vec{r}, \alpha, Q^{2}\right)$ is the LF wave function of the $|q \bar{q}\rangle$ Fock state with transverse separation $\vec{r}$, which depends also on the photon virtuality $Q^{2}$ and the longitudinal momentum fraction $\alpha=p_{q}^{+} / p_{\gamma}^{+}$ of the photon's LF momentum carried by the quark where $\sigma_{q \bar{q}}(\vec{r}, s)$ is the dipole cross section.

Quark shadowing. Assuming the lowest Fock component $|q \bar{q}\rangle$, the effect of the second term in Eq. (2) corresponds to the higher twist quark shadowing. Application of the Green function technique allows controlling precisely the coherence length effects and consequently, the corresponding shadowing dynamics by means of the following formula [4],

$$
\Delta \sigma_{t o t}\left(x_{B j}, Q^{2}\right)=\frac{1}{2} \operatorname{Re} \int d^{2} b \int_{-\infty}^{\infty} d z_{1} \rho_{A}\left(b, z_{1}\right) \int_{z_{1}}^{\infty} d z_{2} \rho_{A}\left(b, z_{2}\right) \int_{0}^{1} d \alpha A\left(z_{1}, z_{2}, \alpha\right),
$$

where

$$
A\left(z_{1}, z_{2}, \alpha\right)=\int d^{2} r_{2} \Psi_{q \bar{q}}^{*}\left(\vec{r}_{2}, \alpha, Q^{2}\right) \sigma_{q \bar{q}}\left(r_{2}, s\right) \int d^{2} r_{1} G_{q \bar{q}}\left(\vec{r}_{2}, z_{2} ; \vec{r}_{1}, z_{1}\right) \sigma_{q \bar{q}}\left(r_{1}, s\right) \Psi_{q \bar{q}}\left(\vec{r}_{1}, \alpha, Q^{2}\right) .
$$

Here $\rho_{A}(b, z)$ represents the nuclear density function defined at the point with longitudinal coordinate $z$ and impact parameter $\vec{b}$. The Green function $G_{q \bar{q}}\left(\vec{r}_{2}, z_{2} ; \vec{r}_{1}, z_{1}\right)$ describes the propagation of the $q \bar{q}$ pair through the nucleus between points with longitudinal coordinates $z_{1}$ and $z_{2}$ and with initial and final transverse separations $\vec{r}_{1}$ and $\vec{r}_{2}$. It satisfies the following two-dimensional Schrödinger equation,

$$
i \frac{d}{d z_{2}} G_{q \bar{q}}\left(\vec{r}_{2}, z_{2} ; \vec{r}_{1}, z_{1}\right)=\left[\frac{\epsilon^{2}-\Delta_{r_{2}}}{2 v \alpha(1-\alpha)}+V_{q \bar{q}}\left(z_{2}, \overrightarrow{r_{2}}, \alpha\right)\right] G_{q \bar{q}}\left(\vec{r}_{2}, z_{2} ; \vec{r}_{1}, z_{1}\right)
$$

with the boundary condition

$$
\left.G_{q \bar{q}}\left(\overrightarrow{r_{2}}, z_{2} ; \vec{r}_{1}, z_{1}\right)\right|_{z_{2}=z_{1}}=\delta^{2}\left(\overrightarrow{r_{1}}-\overrightarrow{r_{2}}\right) .
$$

In (7) the variable $\varepsilon^{2}=m_{q}^{2}+\alpha(1-\alpha) Q^{2}$ and the imaginary part of the LF potential $V_{q \bar{q}}\left(z_{2}, \vec{r}_{2}, \alpha\right)$ describes the attenuation of the $q \bar{q}$ photon fluctuation in the medium and has the following form,

$$
\operatorname{Im} V_{q \bar{q}}\left(z_{2}, \vec{r}, \alpha\right)=-\frac{\sigma_{q \bar{q}}(\vec{r}, s)}{2} \rho_{A}\left(b, z_{2}\right) .
$$

The numerical solution of the corresponding evolution equation for the Green function can be found in [5] and allows to adopt an arbitrary phenomenological model for dipole cross section, as well as the realistic nuclear density function. Nevertheless, when the CL is long enough, $l_{c} \gg R_{A}$ 
at very small Bjorken $x_{B j}$, one can apply the simplified eikonal formula [3] for calculation of quark shadowing.

Gluon shadowing. In the rest frame of the nucleus, it corresponds to the process of gluon radiation and shadowing corrections, related to multiple interactions of the radiated gluons in the nuclear medium. Assuming sufficiently long-lived multi-gluon Fock states of the photon, it is a consequence of the suppression of bremsstrahlung by the interference of radiation from different scattering centres (the Landau-Pomeranchuk effect). The expected kinematic region at planned EICs allows to consider only $q \bar{q} G$ Fock component containing only one gluon since the CL of higher multi-gluon photon fluctuations is too short for the manifestation of the corresponding shadowing corrections. Similar to the quark shadowing case, we apply the Green function technique as well for proper incorporation of realistic lifetimes of gluon fluctuations. We calculate the suppression factor $R_{G}$ given as the ratio of gluon densities in nuclei and nucleon,

$$
R_{G}\left(x_{B j}, Q^{2}\right)=\frac{G_{A}\left(x_{B j}, Q^{2}\right)}{A G_{N}\left(x_{B j}, Q^{2}\right)} \sim 1-\frac{\Delta \sigma_{t o t}(q \bar{q} G)}{A \sigma_{\text {tot }}^{\gamma^{*} N}\left(x_{B j}, Q^{2}\right)},
$$

where the inelastic correction $\Delta \sigma_{t o t}(q \bar{q} G)$ to the total cross section $\sigma_{t o t}^{\gamma^{*} A}\left(x_{B j}, Q^{2}\right)$ reads,

$$
\begin{aligned}
& \Delta \sigma_{t o t}(q \bar{q} G)=\operatorname{Re} \int_{0}^{\infty} d^{2} b \int_{-\infty}^{\infty} d z_{2} \int_{-\infty}^{z_{2}} d z_{1} \rho_{A}\left(b, z_{1}\right) \rho_{A}\left(b, z_{2}\right) \int d^{2} x_{2} d^{2} y_{2} d^{2} x_{1} d^{2} y_{1} \int d \alpha_{q} \frac{d \alpha_{G}}{\alpha_{G}} \\
& \times \quad F_{\gamma^{*} \rightarrow q \bar{q} G}^{\dagger}\left(\vec{x}_{2}, \vec{y}_{2}, \alpha_{q}, \alpha_{G}\right) G_{q \bar{q} G}\left(\vec{x}_{2}, \vec{y}_{2}, z_{2} ; \vec{x}_{1}, \vec{y}_{1}, z_{1}\right) F_{\gamma^{*} \rightarrow q \bar{q} G}\left(\vec{x}_{1}, \vec{y}_{1}, \alpha_{q}, \alpha_{G}\right) .
\end{aligned}
$$

Here variables $\vec{x}$ and $\vec{y}$ represent the transverse distances from the gluon to the quark and antiquark, respectively; $\alpha_{q}$ is the fraction of the LF momentum of the $q \bar{q}$ carried by the quark; $\alpha_{G}$ is the fraction of the photon momentum carried by the gluon; $G_{q \bar{q} G}\left(\vec{x}_{2}, \vec{y}_{2}, z_{2} ; \vec{x}_{1}, \vec{y}_{1}, z_{1}\right)$ is the LF Green function for the $q \bar{q} G$ state; and $F_{\gamma^{*} \rightarrow q \bar{q} G}$, is the amplitudes of diffractive $q \bar{q} G$ production in a $\gamma^{*} N$ interaction.

In (11) the Green function $G_{q \bar{q} G}$ describes the propagation of the three-body system. This requires complicated calculations. However, this can be avoided by suppressing the intrinsic $q \bar{q}$ separation, i.e. assuming $\vec{x}=\vec{y}$. In this case, the Green function essentially simplifies and describes the propagation of a gluon-gluon dipole through a medium. Another simplification of gluon shadowing calculations, without any significant impact on the accuracy of the obtained results, is based on assuming a quadratic form for the dipole cross section, $\sigma_{q \bar{q}}^{N}(r, s)=C(s) r^{2}$. The factor $C(s)$ is obtained from various phenomenological dipole models, and the corresponding differences in predictions of gluon shadowing can be treated as a measure of underlined theoretical uncertainties. The calculational details can be found in [3].

Since the inclusion of higher multi-gluon fluctuations is very complicated, their contribution to gluon shadowing can be effectively taken into account by eikonalization of the calculated factor $R_{G}\left(x_{B j}, Q^{2}\right)$ [6]. This leads to the following renormalisation of the dipole cross section anywhere in expressions for the photoabsorption cross section, $\sigma_{q \bar{q}}^{N}(r, x) \Rightarrow \sigma_{q \bar{q}}^{N}(r, x) \cdot R_{G}\left(x, Q^{2}\right)$.

\section{Predictions for nuclear shadowing}

Figure 1 shows how the nucleus-to-nucleon ratio of the proton structure functions depends on $x_{B j}$ at several fixed values of $Q^{2}$ corresponding to kinematic regions accessible by future 
experiments at EICs. Predictions were calculated using the LF Green function formalism, and various models for dipole cross sections, such as GBW, KST, BGBK and IP-sat [8] depicted by different colors. Consequently, the differences between the onsets of nuclear shadowing represent a measure of theoretical uncertainty. The thick lines correspond to predictions including only higher twist quark shadowing. The boundaries of filled areas represent our results with additional gluon shadowing corrections adopting two different quadratic shapes of $\sigma_{q \bar{q}}(r, s)$ as described in [3], giving so a particular uncertainty arising from the gluon shadowing contribution. The sufficiently precise data from the future EICs will allow to rule out various models for the dipole cross section.

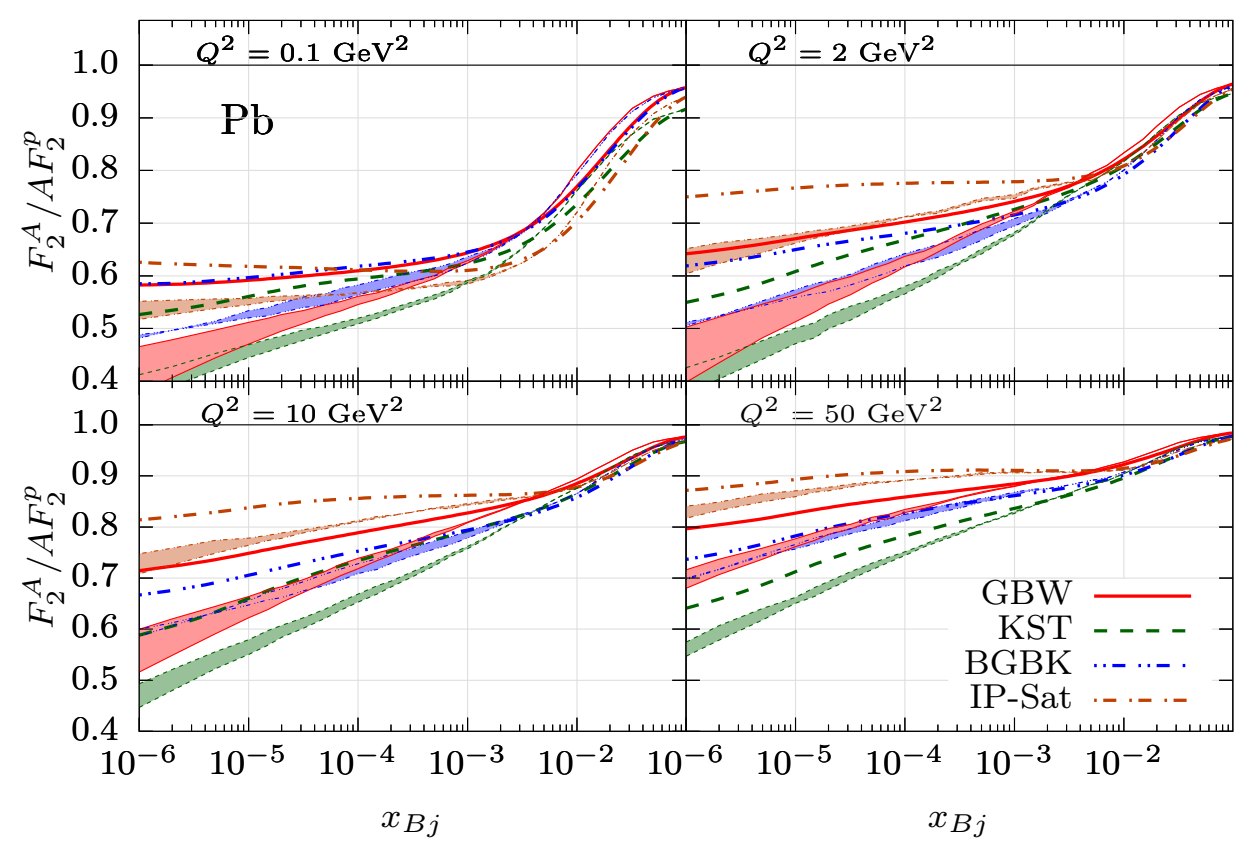

Figure 1: Predictions for the overall nuclear shadowing including the contribution from the lowest $q \bar{q}$ photon fluctuations, as well as from the higher multigluon Fock states $|q \bar{q} G \ldots\rangle$, effectively included via eikonalization of the gluon suppression factor $R_{G}\left(x_{B j}, Q^{2}\right)$. Calculations have been performed for the $\mathrm{Pb}$ target as a function of $x_{B j}$ at different fixed $Q^{2}$. The boundaries of filled areas correspond to contributions from multi-gluon Fock states calculated with different factors $C(s)$ [3]. Predictions were calculated using several dipole models GBW, KST, BGBK and IP-sat [8].

One of the main advantages of the LF color dipole formalism based on the path integral technique inheres in additional power to predict the impact parameter behaviour of shadowing effects. Fig. 2 demonstrates their onset in central, peripheral and minimum bias collisions. Such different manifestation of shadowing effects at various centrality classes can be potentially verified by future measurements at EICs.

\section{Conclusions}

In this report, we adopted the LF Green function formalism for investigation of nuclear shadowing expected by experiments at future EICs. This allows a comprehensive study of coherence effects resulting from destructive interference of the amplitudes for which the interaction takes place on different bound nucleons. We have treated separately shadowing of quarks and gluons repre- 


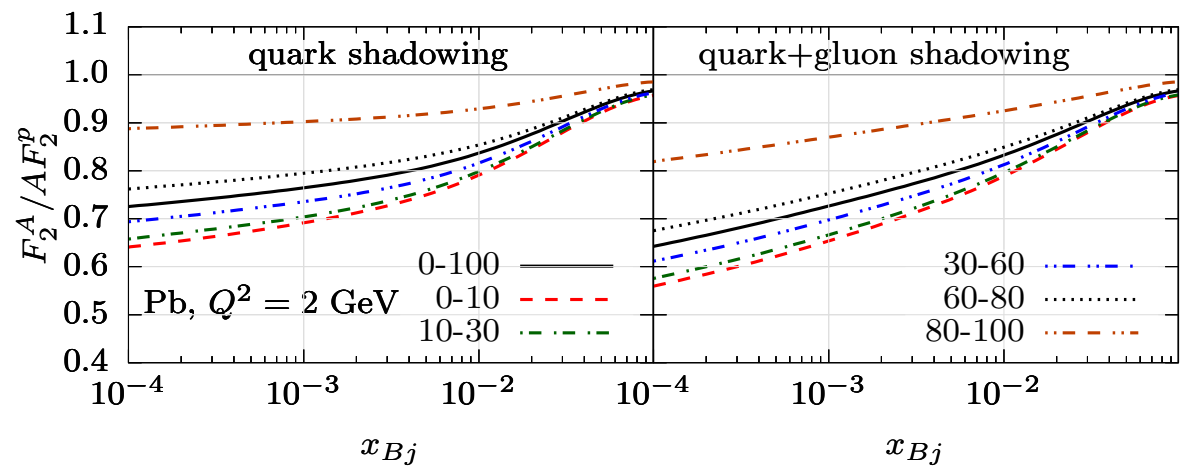

Figure 2: Prediction for the quark shadowing excluding (left panel) and including (right panel) gluon shadowing corrections (right panel) for different centrality classes as function of $x_{B j}$ using the GBW model for the dipole cross section.

senting the higher and leading twist effects related to the lowest $q \bar{q}$ and higher multi-gluon Fock components of the photon, respectively. The later dominates at smaller $x_{B j}$ since corresponding effective masses are larger than for the $|q \bar{q}\rangle$ Fock state, thus requiring much higher photon energies for the manifestation of the maximal onset of gluon shadowing corrections. We demonstrated that predictions for nuclear shadowing at small $x_{B j}$ are strongly correlated with phenomenological models for the dipole cross section used in our analysis. We have also discussed how uncertainties in the estimations of gluon shadowing corrections can affect the final results of the nucleus-to-nucleon ratio of the proton structure functions. We have also found quite a strong dependence of shadowing effects on the nuclear impact parameter. The new precise data from future EICs can shed light on a magnitude of gluon shadowing corrections and can help us to better understand the manifestation of saturation effects at small $x_{B j}$.

Acknowledgment: This work was supported by the project Centre of Advanced Applied Sciences with the number: CZ.02.1.01/0.0/0.0/16-019/0000778 (Czech Republic). Project Centre of Advanced Applied Sciences is co-financed by European Union. The work of J.N. was partially supported by the grant LTT18002 of the Ministry of Education, Youth and Sports of the Czech Republic, and by the Slovak Funding Agency, Grant 2/0007/18.

\section{References}

[1] A. Accardi et al.; Eur. Phys. J. A52, 268 (2016).

[2] J.L. Abelleira Fernandez et al. [LHeC Study Group]; J. Phys. G39, 075001 (2012).

[3] M. Krelina and J. Nemchik, Eur. Phys. J. Plus 135, no.6, 444 (2020).

[4] J. Raufeisen, A.V. Tarasov and O.O. Voskresenskaya, Eur. Phys. J. A 5, 173 (1999).

[5] J. Nemchik; Phys. Rev. C68, 035206 (2003).

[6] B. Kopeliovich, A. Tarasov and J. Hufner; Nucl. Phys. A696, 669 (2001).

[7] L. Zheng, E. C. Aschenauer and J. H. Lee, Eur. Phys. J. A 50, no.12, 189 (2014).

[8] B. Z. Kopeliovich, A. Schafer and A. V. Tarasov, Phys. Rev. D 62, 054022 (2000); K. J. GolecBiernat and M. Wusthoff, Phys. Rev. D 59, 014017 (1998); J. Bartels, K. J. Golec-Biernat and H. Kowalski, Phys. Rev. D 66, 014001 (2002); A. H. Rezaeian, M. Siddikov, M. Van de Klundert and R. Venugopalan, Phys. Rev. D 87, no.3, 034002 (2013). 